Bull. Egypt. Soc. Physiol. Sci. Vol. (41) Issue (3), 410-427

\author{
Bull. of Egyp. Soc. Physiol. Sci. \\ (Official Journal of Egyptian Society for Physiological Sciences) \\ (pISSN: 1110-0842; eISSN: 2356-9514)
}

\title{
Effect of L-Ascorbic Acid and Alpha-Tocopherol on The Monosodium Glutamate- induced Neurobehavioral Changes in Rats
}

\author{
Safaa ME Saleh ${ }^{\mathrm{a}}$, Omnia Ameen ${ }^{\mathrm{a}}$, Marwa S Gad-Allah ${ }^{\mathrm{b}}$, Aya SA Saleh ${ }^{\mathrm{a}^{*}, H_{\text {Hesham AD }}}$ \\ Abdel-Razek ${ }^{\mathrm{a}}$ \\ ${ }^{a}$ Medical Physiology Department, Faculty of Medicine, Menoufia University, Shebein El-Koum, Egypt. \\ ${ }^{b}$ Pathology Department, Faculty of Medicine, Menoufia University, Shebein El-Koum, Egypt.
}

Submit Date: Oct 21, 2020

Accept Date: Jan 11, 2021

Available Online: Feb 1, 2021

\section{Keywords}

monosodium

glutamate

L-ascorbic acid

alpha-tocopherol

GFAP

synaptophysin

\section{Abstract}

Background: Monosodium Glutamate (MSG) is one of the most commonly used flavorenhancing substances that may lead to neurological disorders. Objectives: The present work aimed to evaluate the role of L-ascorbic acid (AA) and $\alpha$-tocopherol $(\alpha \mathrm{T})$ on the MSGinduced memory and neurobehavioral changes in rats. Methods: Thirty male Wistar albino rats were randomized into five equal groups: (1) control group, (2) MSG group received MSG (2mg/g BW) daily, (3) MSG+A group received MSG as in MSG group, and AA (100 $\mathrm{mg} / \mathrm{kg}$ BW) daily, (4) MSG+T group received MSG as in MSG group, and $\alpha \mathrm{T}$ (600 mg/kg BW) twice weekly, and (5) MSG+AT group received MSG as in MSG group, AA as in MSG+A group, and $\alpha \mathrm{T}$ as in MSG+T group. After 3 weeks, neurobehavioral changes were assessed by open field test and Y maze. Oxidative stress markers were estimated, and immunohistochemistry was studied in hippocampal region. Results: MSG resulted in impairment of memory and induction of anxiety, with increased hippocampal malondialdehyde and decreased superoxide dismutase and glutathione peroxidase. Treatment with AA or $\alpha \mathrm{T}$ improved all the measured biochemical parameters, and the MSG-induced hippocampal degenerative changes, with decreased glial fibrillary acidic protein (GFAP) and synaptophysin expression. Combined administration of both vitamins was more effective in amelioration of MSG-induced impairments rather than taking AA or $\alpha \mathrm{T}$ alone. Conclusion: Both AA and $\alpha \mathrm{T}$ exhibit protective effects against neurobehavioral changes, oxidative stress and hippocampal degenerative changes induced by MSG toxicity, with more potent efficacy of their combination.

Corresponding author: Aya SA Saleh, Medical Physiology Department, Faculty of Medicine, Menoufia University, Shebein El-Koum, Egypt. Tel. +02 01092695226. E-mail: saleh.aya33@ yahoo.com 


\section{INTRODUCTION}

Monosodium glutamate (MSG), the sodium salt of glutamic acid, is one of the most commonly used aromas enhancing agents for increasing flavor in meals, and its consumption has recently increased in daily food intake (1). The studies on experimental animals have confirmed toxic effect of MSG in different organs, mainly manifested by increased oxidative stress, cytotoxicity, immunosuppression, reproductive toxicity in both males and females, obesity, asthma, autism and numerous other ailments. MSG is known to produce impairment in memory retention, damage in the hypothalamic neurons, and alterations in mitochondrial lipid peroxidation and antioxidant status in different regions of the brain (2). MSGinduced spatial memory and learning impairment could be due to deficiency of N-methyl-Daspartate (NMDA) glutamate receptors in the hippocampus, degenerative retinal function with impaired vision or cholinergic deficiency (3).

Glial fibrillary acidic protein (GFAP) is one of a family of intermediate filament proteins that serve largely cytoarchitectural function. GFAP expression is considered a sensitive and reliable marker for reactive astrogliosis in response to central nervous system injuries (4). Another marker of synaptic plasticity is synaptophysin that forms about $7 \%$ of the total synaptic vesicle proteins; it is used as a specific protein marker for the presynaptic terminals, and its level is related to the synaptic density. In fact, the synaptic structure is the morphological base of learning and memory processes (5).

The small molecule antioxidants in the body include L-ascorbic acid (AA), the active form of vitamin $\mathrm{C}$, and $\alpha$-tocopherol $(\alpha \mathrm{T})$, the active form of vitamin E (6). AA is a water-soluble antioxidant and a free radical scavenger, which was reported to play an important role in the general oxidationreduction processes (7). $\alpha \mathrm{T}$ is needed for the mitochondria electron transport function; as it donates labile hydrogen to membranes, and in this way, it terminates peroxidation chain reactions by scavenging chain-propagating free radicals (8).

Combinations of two or three antioxidants have led to increase the beneficial outcomes on learning, and to decrease oxidative stress $(9,10)$, when compared with each antioxidant alone. The basic rationale for combining vitamin $\mathrm{E}$ and vitamin $\mathrm{C}$ stems from their different mechanisms and loci of action, and their interaction within a redox network (11). In rodents, the combination of vitamins $\mathrm{C}$ and $\mathrm{E}$ has been shown to prevent homocysteine-induced functional impairments, reduce age-associated impairments in cognitive function, decrease oxidative stress in the brain of old diabetic rats, and protect against intermittent cold exposure-induced oxidative stress in the hypothalamus and cortex of old rats (12). While these studies demonstrated that the combination of the two vitamins is effective in improving brain function and decreasing oxidative stress, most research workers did not systematically compare the stand-alone intervention with their combination, and therefore, did not provide evidences of an additive or synergistic effect.

In the present study, we shed light on the potential effects of AA and $\alpha \mathrm{T}$ on MSG-induced neurobehavioral changes in rats, and the possible underlying mechanisms were studied. 


\section{Material and Methods}

\subsection{Animals and experimental groups.}

Thirty male Wister albino rats, weighing 160-200 g each, and aging 2 - 3 months were obtained from a local animal providing facility. Animals were caged, three per cage, in fully ventilated cages (80 $\mathrm{x} 40 \times 30 \mathrm{~cm}$ ) at normal room temperature under an artificial 12-hours light/dark cycle, with free access to water and semi-synthetic balanced diet. The animal housing, handling, sampling and experimental procedures were performed according to the guide for the care and use of laboratory animals published by the US National Institutes of Health (13). The protocol of the study was approved by The Ethical Committee of Faculty of Medicine, Menoufia University.

After two weeks of acclimatization, rats were divided randomly into five equal groups, 6 rats each: [1] Control (C) group: normal rats were orally given $0.5 \mathrm{ml}$ of distilled water by esophageal gavage, once daily, and were intraperitoneally (i.p.) injected with $0.5 \mathrm{ml}$ of olive oil (Alrayah Company, Egypt), twice weekly, for a duration of three weeks. [2] Non-treated monosodium glutamate-administered (MSG) group: animals were orally given $2 \mathrm{mg} / \mathrm{g}$ body weight (BW) of MSG (Ajinomoto Co. Inc., Tokyo, Japan) (14) dissolved in $0.5 \mathrm{ml}$ of distilled water by esophageal gavage, once daily, and were i.p. injected with olive oil as in control group for a duration of three weeks. [3] L-ascorbic acidtreated monosodium glutamate-administered (MSG+A) group: MSG was orally given as in MSG group, together with $100 \mathrm{mg} / \mathrm{kg} \mathrm{BW}$ of AA (Chemical industries development, Giza, Egypt) (14) dissolved in $0.5 \mathrm{ml}$ of distilled water by esophageal gavage, once daily, and rats were i.p. injected with olive oil as in control group for three weeks. [4] Alpha-tocopherol-treated monosodium glutamate-administered (MSG+T) group: MSG was given as in MSG group, with i.p. injection of $600 \mathrm{mg} / \mathrm{kg}$ BW of $\alpha \mathrm{T}$ (Pharco Pharmaceuticals, Alexandria, Egypt) dissolved in $0.5 \mathrm{ml}$ of olive oil, twice weekly (15) for three weeks. [5] Combined L-ascorbic acid- and $\alpha$-tocopherol-treated monosodium glutamate-administered (MSG+AT) group: MSG and AA were orally given to rats as in MSG and MSG+A groups, respectively, and $\alpha \mathrm{T}$ was i.p. injected as in MSG+T group for three weeks. Animals of all groups survived until the end of the experiment.

\subsection{Study design:}

At the end of the experimental period of three weeks, neurobehavioral assessment of all rats was done using open field and $\mathrm{Y}$ maze tests. Thereafter, rats were anaesthetized by inhalation of $70 \%$ ethanol for 10 minutes, and sacrificed by cervical elongation and dislocation. The skull of each rat was opened, and the brain was extracted and washed with ice-cold phosphate buffer saline (pH 7.4). The left hemisphere was weighed and prepared for hippocampus tissue homogenization to measure the pro-oxidant malondialdehyde (MDA) level and the antioxidant enzymatic activities of glutathione peroxidase (GPx) and superoxide dismutase (SOD) in the brain hippocampus. The right hemisphere was fixed in $10 \%$ formalin saline for histopathological study using Hematoxylin and Eosin staining (H\&E). The expression of synaptophysin and GFAP in hippocampal neurons was evaluated by immunohistochemistry method. 
2.3. Assessment of behavior and spatial memory.

2.3.1. Open field test: Each rat was placed in the center of an open field box $(1 \mathrm{~m} \times 1 \mathrm{~m} \times 50 \mathrm{~cm})$ with a floor divided into squares $(20 \mathrm{~cm} \times 20 \mathrm{~cm})$ units. Rat was put in the center of the field, and locomotor activity was observed for $5 \mathrm{~min}$ in a quiet room illuminated with controlled light. The floor of the box was cleaned with $70 \%$ ethanol between rats. The following parameters were recorded using digital video camera: latent period, squares crossed, center crossing, rearing and grooming (16). Rats were not exposed to prior training to motivate them to explore the box.

\subsubsection{Y-maze:}

Y-maze was constructed using wood and the three arms were made of equal sizes $(60 \mathrm{~cm}$ long, 11.5 $\mathrm{cm}$ width and $25 \mathrm{~cm}$ height). Each rat was placed in the center of the Y-maze to explore the maze for 5 min duration. An entry was scored when the four paws of the animal were completely in the arm of the Y-maze. Alternation was defined as any three consecutive entries of the three different arms; they were counted as a correct choice. Spontaneous alternation refers to the natural tendency of rodents to spontaneously choose alternate arms in a Y-maze, and it is considered a quick and relatively simple test of spatial working memory (17).

The percentage of spontaneous alternation (the ratio of correct choices) was calculated by dividing the total number of alternations by the total number of choices minus 2 (18). The floor of the box was cleaned with $70 \%$ ethanol between rats.

\subsection{Biochemical assays.}

The left half of the rat brain was perfused with PBS solution (Biodiagnostic Company, Egypt), then the hippocampal region was homogenized in $5 \mathrm{ml}$ cold buffer per gram tissue. The hippocampal tissue homogenate was centrifuged at $4000 \mathrm{rpm}$ for 15 min at $4{ }^{\circ} \mathrm{C}$, and the supernatant was removed and stored at $-80{ }^{\circ} \mathrm{C}$ until measurements of the MDA level, and the GPx and SOD enzymatic activities by colorimetric methods using their specific kits purchased from Bio-diagnostics (Giza, Egypt) according to the manufacturer's instruction.

\subsection{Histopathological assessment of the brain} tissue.

2.5.1. Histological examination: The right hemisphere of the brain of each rat was fixed in $10 \%$ formalin solution, embedded in paraffin, and serial coronal sections of $5 \mu \mathrm{m}$ thickness were obtained using a freezing sledge microtome. Sections of brain hippocampus were then stained with Hematoxylin and Eosin (H\&E) for examination with light microscope (19).

\subsubsection{Immunohistochemical assessment: Serial} brain hippocampus sections were cut at $5 \mu \mathrm{m}$ thickness, and were mounted on positively charged glass slides using secondary ultra-vision detection system (Thermo Scientific, USA). Sections were deparaffinized and hydrated, then incubated with hydrogen peroxide blocking solution for $15 \mathrm{~min}$ in humid chambers at room temperature. Slides were washed twice in phosphate buffer, and incubated with pepsin digestive enzyme, and then washed 4 times in the buffer. Ultraviolet block was applied and incubated for $5 \mathrm{~min}$. Thereafter, primary antibodies of GFAP (Thermo Scientific, USA, ready to use $7 \mathrm{ml}$ ) and synaptophysin (Thermo Scientific, USA, 1:60) were applied on the serial sections for $30 \mathrm{~min}$. Sections were washed, and then biotinylated with goat anti-polyvalent 
antibody (secondary antibody) for $10 \mathrm{~min}$, then washed and followed by streptavidin peroxidase for $10 \mathrm{~min}$ and washed again. To develop color reaction, one drop of "DAB Plus" chromogen was added to $2 \mathrm{ml}$ of "DAB Plus" substrate, mixed and applied on tissues for $10 \mathrm{~min}$. Sections were then counterstained with Mayer's hematoxylin. Coverslip was applied using mounting media. Positive reaction appeared as brown color (20). In each of the serial sections, 10 high power fields were examined, and the area \% was measured for both GFAP and synaptophysin expression (21).

\subsection{Statistical analysis.}

The results were analyzed using SPSS computer software package, version 22 (Statistical Package for the Social Science, SPSS Inc., Chicago, Illinois, USA). Data were presented as mean \pm standard deviation (SD). Comparison of quantitative variables between the studied groups was done using one-way analysis of variance (ANOVA) test with Tukey's post hoc test. Pearson correlation coefficient (r) was calculated to access correlation between two continuous variables. Differences were considered statistically significant at $\mathrm{p} \leq 0.05$.

\section{Results}

\subsection{Cognitive and behavioral changes.}

\subsubsection{Performance of open field test:}

The number of squares crossed by the animals of MSG group was significantly decreased, when compared with that of $\mathrm{C}$ group. In $\mathrm{MSG}+\mathrm{A}$, MSG+T and MSG+AT groups, numbers of squares crossed were significantly more than that of MSG group, but still significantly less than that of $\mathrm{C}$ group. Also, number of squares crossed in MSG+AT group was significantly higher than those of MSG+A and MSG+T groups. There was insignificant variation between MSG+A and MSG+T groups.

The numbers of rearing and grooming were significantly increased in MSG group versus C group. In MSG+A, MSG+T and MSG+AT groups, numbers of rearing and grooming were significantly reduced compared to MSG group, but still significantly more than those of $\mathrm{C}$ group. Also, rearing and grooming in animals of MSG+AT group were significantly lower than those of MSG+A and MSG+T groups. There was insignificant difference between MSG+A and MSG+T groups.

Notably, there were insignificant differences between all groups regarding latent period and center crossing. Table (1) demonstrates the performance of open field test by all the studied groups.

\subsubsection{Performance of $Y$-Maze.}

The percentage of spontaneous alternations was significantly decreased in MSG group, when compared with that of $\mathrm{C}$ group. In $\mathrm{MSG}+\mathrm{A}$, MSG+T and MSG+AT groups, percentages of spontaneous alternations were significantly higher than that of MSG group, but still significantly less than that of $\mathrm{C}$ group. Also, the percentage of spontaneous alternations of MSG+AT group showed a significant increase compared to those of MSG+A and MSG+T groups. There was insignificant difference between values of MSG+A and MSG+T groups (Table 1). 
Table (1): Performance of open field test and percentage of spontaneous alteration in $Y$-maze test in control (C) group, non-treated monosodium glutamate-administered (MSG) group, and monosodium glutamate-administered groups treated with L-ascorbic acid (MSG+A), $\alpha$-tocopherol (MSG+T) or both (MSG+AT).

\begin{tabular}{|c|c|c|c|c|c|}
\hline $\begin{array}{l}\text { Group } \\
\text { Parameter }\end{array}$ & $\mathrm{C}$ & MSG & $\mathrm{MSG}+\mathrm{A}$ & $\mathrm{MSG}+\mathrm{T}$ & $\mathrm{MSG}+\mathrm{AT}$ \\
\hline Latent period & $1.65 \pm 0.41$ & $1.59 \pm 0.44$ & $1.63 \pm 0.50$ & $1.66 \pm 0.47$ & $1.67 \pm 0.41$ \\
\hline Center crossing & $1.62 \pm 0.54$ & $1.37 \pm 0.61$ & $1.57 \pm 0.52$ & $1.57 \pm 0.51$ & $1.60 \pm 0.55$ \\
\hline Squares crossed & $64.83 \pm 9.63$ & $24.17 \pm 3.17^{*}$ & $42.67 \pm 6.37$ *\# & $44.17 \pm 6.82 * \#$ & $55.67 \pm 7.60 * \# \$ \Omega$ \\
\hline Rearing & $1.50 \pm 0.27$ & $12.00 \pm 2.10^{*}$ & $7.33 \pm 1.21 * \#$ & $8.67 \pm 1.45^{* \#}$ & $2.67 \pm 0.47 * \# \$ \Omega$ \\
\hline Grooming & $1.67 \pm 0.32$ & $7.83 \pm 1.37 *$ & $3.67 \pm 0.52 * \#$ & $4.00 \pm 0.89 * \#$ & $2.83 \pm 0.58 * \# \$ \Omega$ \\
\hline $\begin{array}{l}\text { Spontaneous } \\
\text { alteration \% }\end{array}$ & $94.7 \pm 3.88$ & $53.3 \pm 5.8^{*}$ & $71.8 \pm 4.91^{\text {*\# }}$ & $72.5 \pm 4.89^{*} \#$ & $85.5 \pm 5.45^{*} \# \$ \Omega$ \\
\hline
\end{tabular}

Data are expressed as mean \pm standard deviation. Number of rats in each group was $\operatorname{six}(\mathrm{n}=6)$. One-way analysis of variance with Tukey's post-hoc test was applied, and significant level was set on $P \leq 0.05$. The marks *, \#, $\$$ and $\Omega$ indicate significant differences, when values are compared with the corresponding values of C, MSG, MSG+A and MSG+T groups, respectively.

\subsection{Changes of oxidative stress markers.}

Table (2) shows the changes of MDA level and enzymatic activities of SOD and GPx in the brain tissue homogenates of the different studied groups. MDA level in MSG group showed a significant elevation, when compared with that in $\mathrm{C}$ group. In $\mathrm{MSG}+\mathrm{A}, \mathrm{MSG}+\mathrm{T}$ and $\mathrm{MSG}+\mathrm{AT}$ groups, MDA levels were significantly decreased, when compared with that of MSG group. MDA level of MSG+AT group was significantly lower than those of MSG+A and MSG+T groups, but still significantly more than that of $\mathrm{C}$ group. There was insignificant variation of MDA levels between MSG+A and MSG+T groups.

The activities of both SOD and GPx antioxidant enzymes were significantly reduced in MSG group, when compared with those of $\mathrm{C}$ group. In MSG+A, MSG+T and MSG+AT groups, SOD and GPx activities were significantly higher than those of MSG group. SOD and GPx activities were significantly increased in MSG+AT group versus $\mathrm{MSG}+\mathrm{A}$ and MSG+T groups. There was a nonsignificant variation of SOD and GPx activities between MSG+A and MSG+T groups.

Table (2): Malondialdehyde (MDA) levels and the enzymatic activities of superoxide dismutase (SOD) and glutathione peroxidase (GPx) in the brain hippocampus tissue homogenates of control (C) group, non-treated monosodium glutamate-administered (MSG) group, and monosodium glutamateadministered groups treated with L-ascorbic acid (MSG+A), $\alpha$-tocopherol (MSG+T) or both (MSG+AT).

\begin{tabular}{lccccc}
\hline \multicolumn{1}{c}{ Group } & $\mathrm{C}$ & MSG & MSG+A & MSG+T & MSG+AT \\
\hline $\begin{array}{l}\text { Parameter } \\
\text { (nmol/g. tissue) }\end{array}$ & $14.70 \pm 2.49$ & $108.33 \pm 6.94 *$ & $59.83 \pm 3.19 * \#$ & $71.00 \pm 8.16 * \#$ & $25.07 \pm 3.22 * \# \$ \Omega$ \\
$\begin{array}{l}\text { SOD } \\
\text { (U/g. tissue) }\end{array}$ & $98.83 \pm 1.94$ & $31.36 \pm 3.90 *$ & $73.49 \pm 10.52 * \#$ & $77.45 \pm 5.23 * \#$ & $87.40 \pm 2.25 * \# \$ \Omega$ \\
$\begin{array}{l}\text { GPx } \\
\text { (U/g. tissue) }\end{array}$ & $83.20 \pm 2.71$ & $28.06 \pm 5.81 *$ & $51.52 \pm 6.21 * \#$ & $52.75 \pm 5.59 * \#$ & $71.38 \pm 7.39 * \# \$ \Omega$ \\
\hline
\end{tabular}

Data are expressed as mean \pm standard deviation. Number of rats in each group was six $(n=6)$. One-way analysis of variance with Tukey's post-hoc test was applied, and the significant level was set on $\mathrm{P} \leq 0.05$. The marks $*, \#, \$$ and $\Omega$ indicate significant differences, when values are compared with the corresponding values of $\mathrm{C}, \mathrm{MSG}, \mathrm{MSG}+\mathrm{A}$ and $\mathrm{MSG}+\mathrm{T}$ groups, respectively. 


\subsection{Histopathological examination.}

\subsubsection{Hematoxylin and eosin stained sections.}

In the H\&E stained sections from hippocampi of the rat brains of $\mathrm{C}$ group, normal appearance of the histological picture was found, with almost organized pyramidal cell layer and unremarkable shrunken cells. The granular cell layer was of normal appearance and cells showed no vacuoles. The molecular layer showed normal neurons, nerve fibers and astrocytes without any histopathological changes (Fig.1[A]). MSG group revealed marked degenerative changes, mainly in pyramidal cell layer; most of cells appeared shrunken with darkly stained cytoplasm and deeply pyknotic nuclei, and were surrounded by a peripheral halo. The nerve fibers were widely separated. These changes were observed in all parts of hippocampus, mainly in the pyramidal cell layer and in the granular cell layer of the dentate gyrus (Fig.1[B]). In MSG+A group, there was moderate improvement of the histological picture, where some pyramidal cells appeared with vesicular rounded nuclei and acidophilic cytoplasm, but still other cells showed deeply stained cytoplasm and pyknotic nuclei (Fig.1[C]). In MSG+T group, there was mild improvement of the histological picture; although most pyramidal cells still showed degenerative changes, other cells appeared with acidophilic cytoplasm and vesicular nuclei (Fig.1[D]). The MSG+AT group showed a good improvement of the histological picture, where most neurons of pyramidal cell layer were quite normal having acidophilic cytoplasm and vesicular nuclei, while only few cells were still showing evidence of degeneration in the form of shrunken cytoplasm and pyknotic nuclei (Fig. $1[\mathrm{E}])$.

\subsubsection{Immunohistochemistry studies.}

Quantitative studies by area \% for GFAP and synaptophysin were demonstrated in Fig. (2) and Fig. (3), respectively. Significant increases in the area \% of both GFAP and synaptophysin expressions were observed in MSG, MSG+A, MSG+T and MSG+AT groups, when compared with those of $\mathrm{C}$ group. Significant decreases of area $\%$ of both GFAP and synaptophysin immunostaining were detected in $\mathrm{MSG}+\mathrm{A}$, $\mathrm{MSG}+\mathrm{T}$ and MSG+AT groups compared to those of MSG group. MSG+AT group showed lower area \% of both GFAP and synaptophysin immunostaining than those of MSG+A and MSG+T groups; however, values of MSG+AT were still significantly more than those of $\mathrm{C}$ group. A nonsignificant variation in area $\%$ of both GFAP and synaptophysin between MSG+A and MSG+T groups was observed. 
[A]

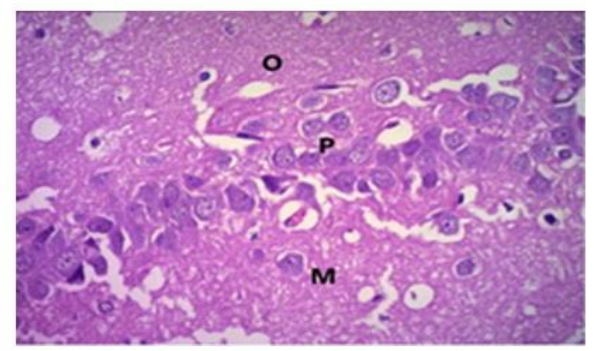

[C]

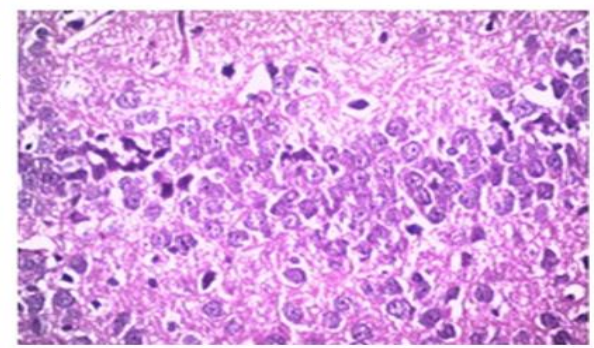

[E]

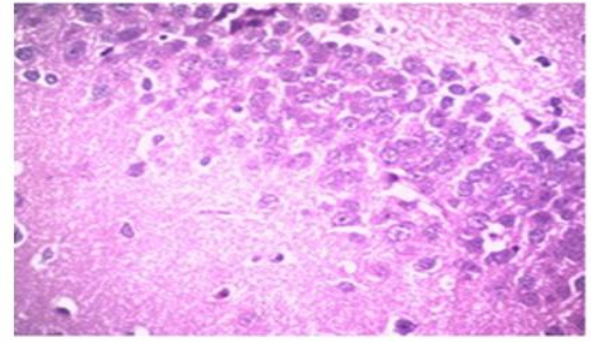

[A]

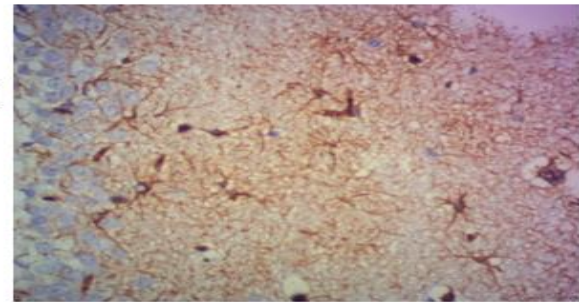

[C]

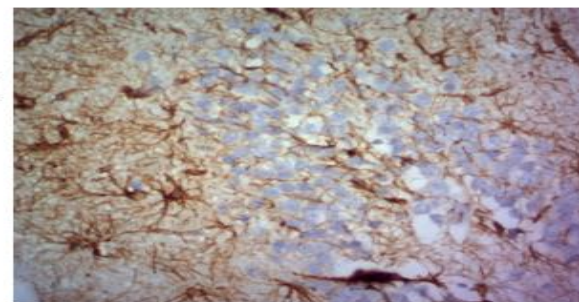

[E]

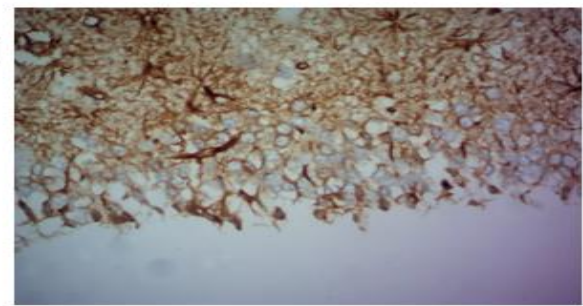

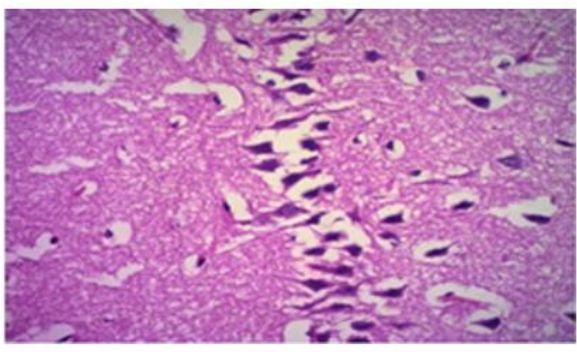

[B]

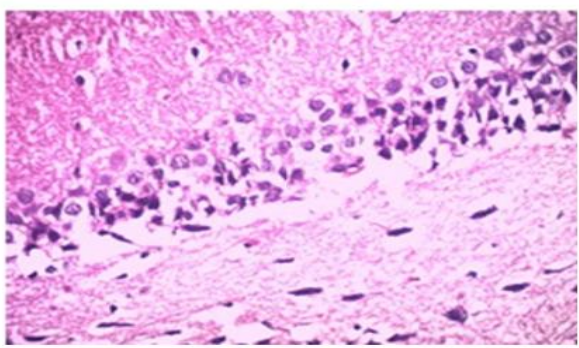

Fig. (1): Hematoxylin and eosin stained sections of hippocampus showing normal architecture in control group $[A]$, neurodegenerative changes in nontreated monosodium glutamate administered group $[B]$, and their amelioration in monosodium glutamateadministered groups treated with Lascorbic acid $[C], \alpha$-tocopherol $[D]$ or both $[\mathbf{E}]$. P: pyramidal cell layer. $\mathrm{O}$ : polymorphic cells. M: molecular cell layer.
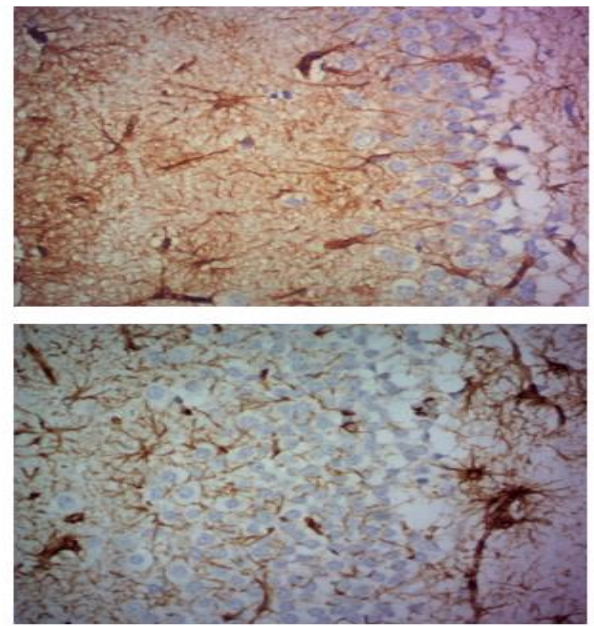

[D]

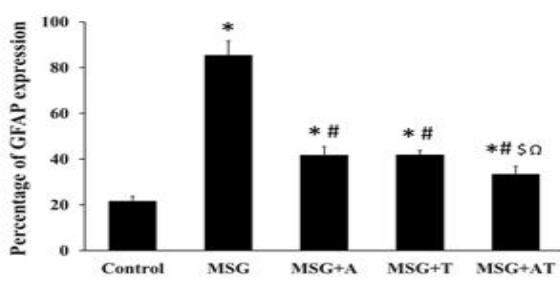

Fig. (2): Glial fibrillary acidic protein (GFAP) immunostaining of hippocampus showing normal expression in control group [A], increased expression in non-treated monosodium glutamateadministered group (MSG, [B]), and its amelioration in monosodium glutamate-administered groups treated with L-ascorbic acid (MSG + , [C]), $\alpha$-tocopherol (MSG +T, [D]) or both (MSG+AT, [E]). Panel [F] represents the percentage of GFAP expression in the above-mentioned groups. Number of rats in each group was $\operatorname{six}(n=6)$. Data are expressed as mean \pm standard deviation. One-way analysis of variance with Tukey's post-hoc test was applied, and the significant level was set on $P \leq 0.05$. The marks *, $\#, \$$ and $\Omega$ indicate a significant variation, when values are compared with control, $M S G, M S G+A$ and $\mathrm{MSG}+\mathrm{T}$ groups, respectively. 
[A]

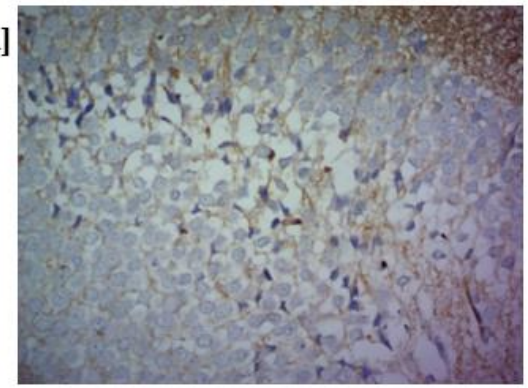

[C]

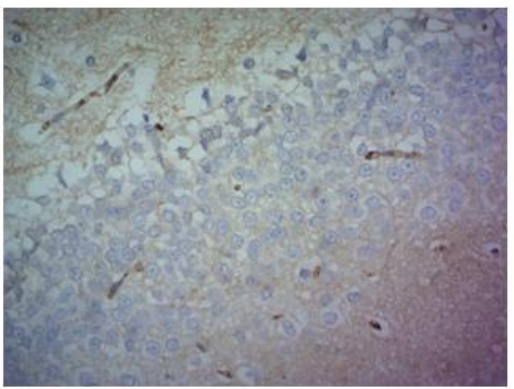

$[\mathbf{E}]$

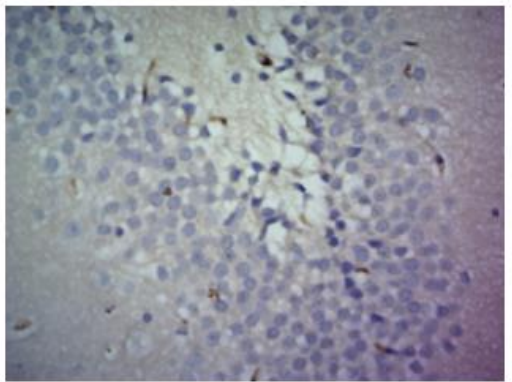

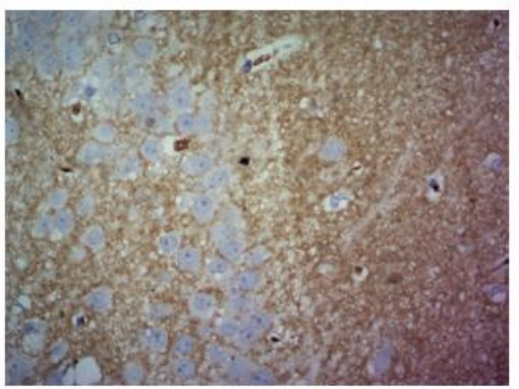
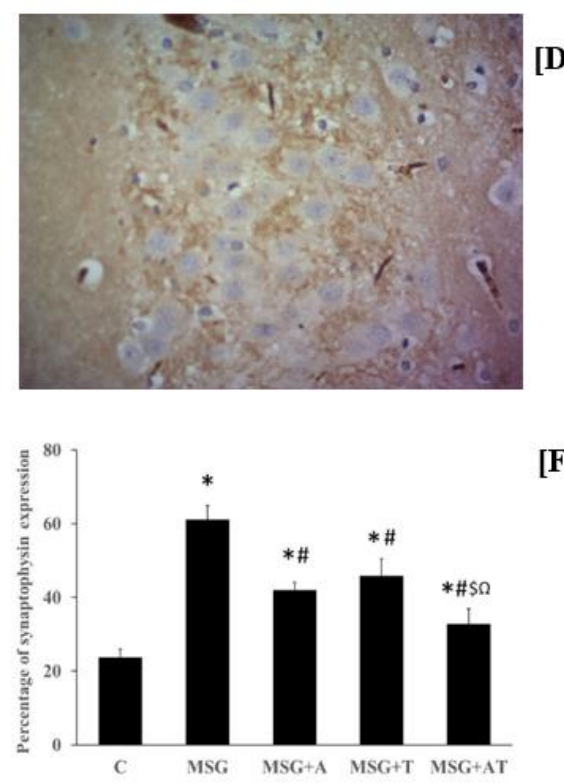

Fig. (3): Synaptophysin immunostaining of hippocampus showing normal expression in control group [A], increased expression in non-treated monosodium glutamate-administered group (MSG, [B]), and its amelioration in monosodium glutamate-administered groups treated with $L$ ascorbic acid (MSG+A, [C]), $\alpha$-tocopherol (MSG+T, [D]) or both (MSA+AT, [E]). Panel [F] represents the percentage of synaptophysin in the above-mentioned groups. Number of rats in each group was six $(n=6)$. Data were expressed as mean \pm standard deviation. One-way analysis of variance with Tukey's post-hoc test was applied, and the significant level was set on $\mathrm{P} \leq 0.05$. The marks *, \#, \$ and $\Omega$ indicate a significant variation, when values are compared with control, MSG, $\mathrm{MSG}+\mathrm{A}$ and $\mathrm{MSG}+\mathrm{T}$ groups, respectively.

\subsection{Correlation between hippocampal}

\section{MDA level and other measured variables.}

Fig. 4 shows the Pearson correlations between the oxidative stress and lipid peroxidation marker, MDA and all the other measured variables in all the studied groups $(n=30)$.

Significant positive correlations were observed between the brain hippocampal MDA level and the latent period $(\mathrm{r}=0.441, \mathrm{P}=0.015)$, mean number of rearing $(\mathrm{r}=0.933, \mathrm{P}=0.000)$, grooming $(\mathrm{r}=0.888$, $\mathrm{P}=0.000$ ) of open field test, and percent area of expression of GFAP $(\mathrm{r}=0.817, \quad \mathrm{P}=0.000)$ and synaptophysin $\quad(\mathrm{r}=0.718, \quad \mathrm{P}=0.000) \quad$ in immunohistochemistry study. On the contrary, significant negative correlations were found between the MDA level and the percentage of spontaneous alternations in $\mathrm{Y}$ maze $(\mathrm{r}=-0.888$, $\mathrm{P}=0.000$ ), number of squares crossed in open field test $(\mathrm{r}=-0.836, \mathrm{P}=0.000)$, and the enzymatic activities of SOD $(r=-0.920, P=0.000)$ and $\mathrm{GPx}$ $(\mathrm{r}=-0.949, \mathrm{P}=0.000)$. No significant correlation was observed between MDA and center crossing in open field test. 

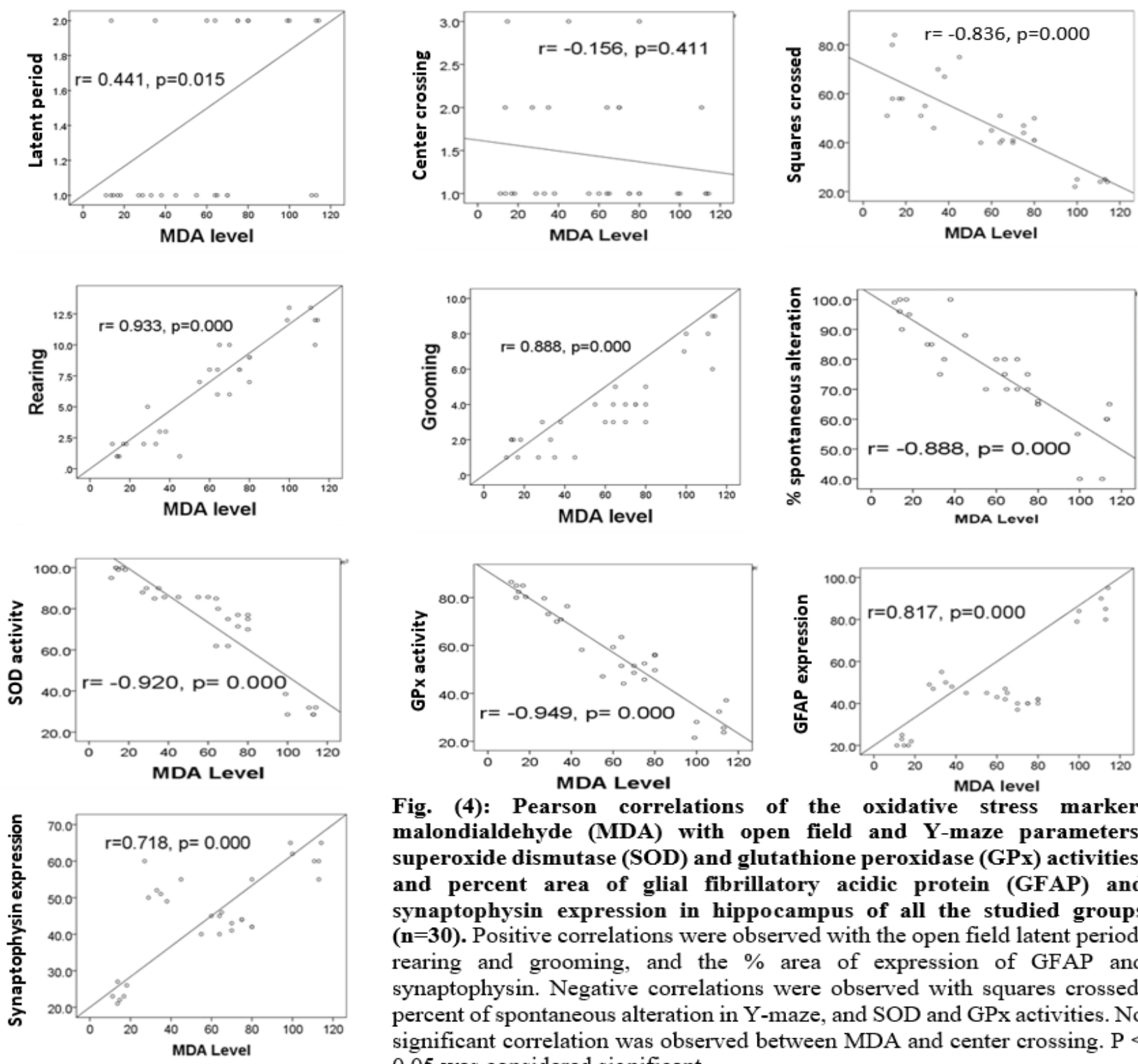

Fig. (4): Pearson correlations of the oxidative stress marker, malondialdehyde (MDA) with open field and $Y$-maze parameters, superoxide dismutase (SOD) and glutathione peroxidase (GPx) activities, and percent area of glial fibrillatory acidic protein (GFAP) and synaptophysin expression in hippocampus of all the studied groups $(\mathbf{n}=\mathbf{3 0})$. Positive correlations were observed with the open field latent period, rearing and grooming, and the \% area of expression of GFAP and synaptophysin. Negative correlations were observed with squares crossed, percent of spontaneous alteration in Y-maze, and SOD and GPx activities. No significant correlation was observed between MDA and center crossing. $\mathrm{P}<$ 0.05 was considered significant.

\section{Discussion}

The present study showed a reduction in the rat cognitive function, as demonstrated by a decrease of percentage of spontaneous alternations in $\mathrm{Y}$ maze in rats given MSG compared to control animals, indicating impairment of working memory, which confirms earlier investigations by Sreejesh and Sreekumaran (22) applying T-maze, and Hassaan et al. (23) working on mice. Their results of open field test were also in consistency with our findings that MSG administration affects locomotor activity through a decrease in the number of squares crossed and an increase in the anxiety-like behavior of rearing and grooming, when compared with normal animals. The impairment of memory and anxiety could be attributed to the excitotoxicity of MSG, as it alters the activity and sensitivity of rat hypothalamicpituitary-adrenocortical axis (24). Also, the accumulated excessive amounts of glutamate by MSG ingestion may act as a potent neurotoxin through activation of proteolytic enzymes. The excitotoxic effect of glutamate is mediated by an interaction with NMDA receptors leading to an uncontrollable rise in intracellular $\mathrm{Ca}^{2+}$ concentration, which activates various enzymes 
contributing to cell death by various mechanisms (3). Also, inhibition of cholinergic system and induction of oxidative stress may mediate MSG neurotoxicity $(3,24)$. On the contrary of our finding, some studies reported that MSG administration either did not affect memory and behavior or increased them slightly $(3,25)$. This contradictory results with what has been obtained in this study may be explained by their usage of lower doses of MSG or performing their experiment for shorter durations.

Our results showed an increase in MDA level and a decrease in SOD and GPx activities in the brain hippocampus of MSG-administered rats, when compared with those of normal rats; a finding that was also reported by previous investigations $(26,27)$. In fact, the brain is particularly vulnerable to membrane lipid peroxidation due to the relatively high abundance of polyunsaturated fatty acids (28). High amount of glutamate excites the glutamate receptors and hyper-activates $\mathrm{Ca}^{2+}$ to enter neurons and cascade towards inside the cell that leads to activation of many hydrolytic enzymes (29). These enzymes injure the cellular structures, such as the cytoskeleton, membranes, mitochondria and DNA, increasing of peroxidation stress and production of free radicals, and finally leading to cell apoptosis $(29,30)$. In order to scavenge ROS, different defense systems exist in the brain, such as enzymatic (SOD, GPx and catalase), nonenzymatic (glutathione and uric acid) and dietary antioxidants. If ROS are not effectively eliminated, they can cause peroxidation of cell membrane phospholipids, proteins and DNA (31). Decreased SOD and GPx activities may be due to their overconsumption or inactivation by production of excessive free radicals, which in turn generate hydroxyl radicals resulting in initiation and propagation of lipid peroxidation (32). Staining of hippocampus of MSG-administered rats by $\mathrm{H} \& \mathrm{E}$ revealed degenerative changes in neurons mainly of pyramidal cell layer and granular cell layer of dentate gyrus. These changes were in the form of shrinkage in the cytoplasm and deeply stained pyknotic nuclei with wide separation of neuropil fibers; changes that come in consistency with previous studies $(3,33)$. Glutamate-induced neuronal death has been reported (29) to be mediated by both apoptotic and necrotic mechanisms, with excitotoxic and oxidative injury, as two distinct pathways by which neuronal death is induced.

GFAP immune staining of hippocampi of MSGadministered rats versus control animals revealed significant increase in $\%$ of the area of GFAP expression in soma of astrocytes and their processes, indicating that MSG causes massive activation of these cells, leading to gliosis of the brain tissue, a finding that was in agreement with previous studies $(34,35)$. Glial cells react to neuronal damage, resulted from physical or chemical insults, by overexpression of GFAP in a trial to protect neurons through the uptake of excitotoxic glutamate, and the production of glutathione and neuroprotective adenosine (36).

On the other hand, Tantawi and Namshan (37) reported that, oral MSG administration to mice, in doses of $2 \mathrm{mg} / \mathrm{kg}$ and $4 \mathrm{mg} / \mathrm{kg}$, showed fragmentation and weak neuronal and GFAP reaction in cerebellum of mice. They explained this by the death of astrocytes due to excess glutamate (37). The discrepancy between our results and this study may be due to the difference in species of rodents or in the dose of MSG used for model 
induction. Synaptophysin immune staining of hippocampus in MSG group revealed an increase in its expression at many synaptic sites when compared with normal animals. This finding was supported by El-Gohari and his colleagues (38), who found that rat retinal synaptophysin immunostained sections became heavily stained after glutamate. El-Tantawi and El-Namshan (37) also proved an increase in synaptophysin immunostaining in cerebellum of MSGadministered mice compared to controls. Nakamura et al. (39) declared that increased synaptophysin expression may be due to compensatory increase in presynaptic terminals density at first stage of degeneration caused by excitotoxic effect of MSG and this was clearly obvious in our results. In contrast, Sasaki-Hamada and colleagues (40) reported that intraperitoneal injection of the MSG did not change the expression level of synaptophysin in rat hippocampus. The absence of effect of increased glutamate on synaptophysin in their study may be due to variations in duration and different routes of MSG administration.

Data of the present work indicated that treatment of MSG-administered rats with AA or $\alpha \mathrm{T}$ improved working memory and reduced anxiety. This was clearly manifested by increasing of the percentage of spontaneous alternation, and decreasing of anxiety-like behaviors (rearing and grooming) with improvement of locomotor activity, when compared with those of MSG-administered animals that did not receive any treatment. However, the percentage of spontaneous alternations was still significantly lower, and rearing and grooming were still significantly higher than normal values. This observation was in agreement with previous studies $(14,41,42)$. Notably, there was no significant difference between $\mathrm{AA}$ and $\alpha \mathrm{T}$ administration on their effect on MSG-administered rats. The neuroprotective effects of each of these vitamins against MSG-induced neurological changes may, at least partially, be due to their potent antioxidant activities, as they were associated with a marked decrease of MDA level and increases of SOD and GPx activities. Still, there were non-significant variations of MDA levels and the SOD and GPx activities between MSG-administered animals treated with AA and $\alpha \mathrm{T}$. The ability of AA to ameliorate the MSG-associated increase in MDA level could be mainly attributed to its scavenging activity towards reactive oxygen species, and thereby reducing the rate of lipid peroxidation and protecting the DNA, proteins or lipids as well as sparing endogenous antioxidant enzymes (43). These results were in harmony with many other researches (41-46). Beside the antioxidant property of AA, its neuroprotective role may also be due to involvement in presynaptic reuptake of glutamate, which inhibits binding of glutamate to the NMDA receptor (47). The obvious improvement in MSGadministered rats treated with $\alpha \mathrm{T}$ could be attributed to its antioxidant effect (48). Lipid peroxidation is minimized by $\alpha \mathrm{T}$ through its ability to transfer its phenolic hydrogen to peroxyl free radical of peroxidized polyunsaturated fatty acids (49).

In our work, $H \& E$ staining of hippocampus of MSG-administered rats treated with AA revealed moderate improvement of the histological picture compared to non-treated ones. Some pyramidal cells appeared with vesicular rounded nuclei and acidophilic cytoplasm, but still other cells showed deeply stained cytoplasm and pyknotic nuclei. These findings were in consistency with those 
reported by Hashem et al. (50) and Ibrahim et al. (51). The protective role of AA in partial preservation of the structure of hippocampus was explained by its antioxidant function, and this finding goes hand in hand with our biochemical results, indicating the potent antioxidant property of AA in the brain. In parallel, the MSGadministered animals treated with $\alpha \mathrm{T}$ revealed mild to moderate improvement of the histological picture, when compared with the MSGadministered rats which did not receive any treatment. Although most pyramidal cells still showing degenerative changes, other cells appeared with acidophilic cytoplasm and vesicular nuclei. This partial correction of the histological structural abnormality of hippocampus by $\alpha \mathrm{T}$ was also reported by other research workers $(46,52)$.

A decrease in the MSG-induced GFAP overexpression in rat hippocampus was observed after treatment with AA. In line, Ibrahim et al. (51) proved a partial protective effect of AA against the degenerative changes induced by MSG through a decrease in gliosis. In our hands, there was significant improvement in the expression of synaptophysin after treatment of MSGadministered rats with $\mathrm{AA}$; a finding that was explained by AA prevention of the degenerative changes of synapses induced by MSG (53).

Similarly, immunohistochemical study revealed significant decline in the percentages of expression of GFAP and synaptophysin in MSG-administered rats when treated with $\alpha \mathrm{T}$. However, GFAP and synaptophysin expressions were still significantly higher than their expression in normal animals. These results assure the protective antioxidant effects of $\alpha \mathrm{T}$ against MSG toxicity previously reported by Ambrogini and his colleagues (54).
The hallmark of the present study is that the combined treatment of MSG-administered rats with AA or $\alpha \mathrm{T}$ resulted in significant better improvements in all measured neurobehavioral, biochemical and histological parameters than isolated treatment with each one of the two vitamins alone. In line with our finding, Shetty and his colleagues (10) stated that administration of two or three antioxidants has led to increased beneficial outcomes on learning and to decreased oxidative stress when compared to each antioxidant alone.

In line with our findings, Farombi and Onyema (32) reported that food substances rich in antioxidants, such as vitamin $\mathrm{C}$, vitamin $\mathrm{E}$ and quercetin significantly modulate the toxic responses resulting from diets containing MSG. Also, Rendon-Ramirez et al. (55) reported that vitamin $\mathrm{C}$ combined with vitamin $\mathrm{E}$ was effective in reducing the MDA level, the marker of lipid peroxidation, in lead-exposed workers. Combined intake of vitamin $\mathrm{C}$ and vitamin $\mathrm{E}$, for at least ten years, was found to help maintaining cognitive functions of women in their 70's (56).

The current observation of the beneficial effect of concomitant use of $\mathrm{AA}$ and $\alpha \mathrm{T}$ in treatment of the MSG-induced disorders may reflect an additive or a synergistic effect of the combination of both vitamins in amelioration of neurobehavioral impairment and oxidative stress induced by MSG, that is possibly achieved by different mechanisms of actions and their interaction. Buettner reported that the basic rationale for combining vitamin $\mathrm{E}$ and vitamin $\mathrm{C}$ stems from their different mechanisms and loci of action, and their interaction within a redox network as $\alpha \mathrm{T}$ is found in all biological membranes and is involved in a chain- 
breaking mechanism to prevent further lipid peroxidation by scavenging peroxyl radicals. In the process, $\alpha \mathrm{T}$ becomes oxidized to $\alpha$-tocopheroxyl radical, which can in turn become very damaging. Based on redox potential and availability of the radical located at the membrane water interface and ascorbate can reduce back the tocopheroxyl radical to tocopherol. Ascorbate is then oxidized and is recycled with the help of enzyme systems using NADH or NADPH (11).

Regarding Pearson correlation studies, the levels of the oxidative stress and lipid peroxidation marker, MDA in hippocampus were found to be positively correlated with latent period, rearing and grooming in open field test and the percentage of hippocampal GFAP and synaptophysin expression. MDA levels were negatively correlated with number of squares crossed, percentage of spontaneous alternations in Y-maze, and the activities of hippocampal SOD and GPx. These correlations come in concordance with the hypothesis which suggests that increased oxidative stress could be a main contributing factor to the impairment of the cognitive and psychomotor performance, associated or not with aging process, in various behavioral tasks $(57,58)$.

\section{Conclusion}

Both AA and $\alpha \mathrm{T}$ provide neuroprotective role against MSG-induced neurobehavioral disturbance, hippocampal structural degenerative changes and oxidative stress, with a more potent protective effect of their combination, possibly by a synergistic effect. Further studies are needed to investigate the effect of the combined treatment of the two studied and other antioxidant vitamins in amelioration of the MSG-induced neurotoxicity in humans. The markers of oxidative stress could also be considered for evaluating therapeutic strategies of MSG-induced neurotoxicity and consequent behavioral alteration.

\section{Acknowledgement}

The authors are grateful to Prof. Dr. Magda Ahmed Mansour, Professor of Histology and Cell Biology, Faculty of Medicine, Menoufia University, Egypt, for her sincere guidance in the interpretation of the histological studies.

Conflict of Interest: Non declared.

Funding: No funding sources.

\section{References}

\section{Yonden Z, Ozcan O, Cimen AY, Delibas}

$\mathbf{N}$. The effect of monosodium glutamate and aspartame on rat hippocampal N-methyl-Daspartate receptor subunits and oxidative stress biomarkers. Int J Clin Exp Med. 9:1864-70, 2016.

2. Sharma V, Deshmukh R. Ajimomoto (Msg): a Fifth Tatse or a Bio Bomb, EJPMR. 2:381-400, 2015.

\section{Onaolapo OJ, Onaolapo AY, Akanmu} MA, Gbola O. Evidence of alterations in brain structure and antioxidant status following 'low-dose' monosodium glutamate ingestion. Pathophysiology. 23:147-56, 2016.

4. Sofroniew M V, Vinters H V. Astrocytes: biology and pathology. Acta Neuropathol. 119:7-35, 2010.

5. Coleman P, Federoff H, Kurlan R. A focus on the synapse for neuroprotection in Alzheimer disease and other dementias. Neurology. 63:1155-62, 2004. 
6. Manna SK, Zhang HJ, Yan T, Oberley LW, Aggarwal BB. Overexpression of manganese superoxide dismutase suppresses tumor necrosis factor-induced apoptosis and activation of nuclear transcription factor- $\kappa \mathrm{B}$ and activated protein-1. J Biol Chem. 273:13245-54, 1998.

7. Sharma J, Solanki D, Solanki M. Amelioration of fluoride toxicity in rats through vitamins (C, D) and calcium. Toxicol Int. 15:111-116, 2008.

8. Halliwell B. Oxidants and the central nervous system: some fundamental questions. Is oxidant damage relevant to Parkinson's disease, Alzheimer's disease, traumatic injury or stroke? Acta Neurol Scand. 80:23-33,1989.

9. Mcdonald SR, Sohal RS, Forster MJ. Concurrent administration of coenzyme Q10 and $\alpha$-tocopherol improves learning in aged mice. Free Radic Biol Med. 38:729-36, 2005.

10. Shetty RA, Ikonne US, Forster MJ, Sumien N. Coenzyme Q10 and $\alpha$ tocopherol reversed age-associated functional impairments in mice. Exp Gerontol. 58:208-18, 2014.

11. Buettner GR. The pecking order of free radicals and antioxidants: lipid peroxidation, alpha-tocopherol, and ascorbate. Arch Biochem Biophys. 300:535-43, 1993.

12. Mock JT, Chaudhari K, Sidhu A, Sumien N. The influence of vitamins E and $\mathrm{C}$ and exercise on brain aging. Exp Gerontol. 94:69-72, 2017.
13. Garber JC, Barbee RW BJ et al. NR. Guide for the care and use of laboratory animals: Institute for laboratory animmal research, division on earth and life studies. washington DC, USA: National Academies Press; eighth. 220 p, (2001).

14. Narayanan SN, Kumar RS, Paval J, Nayak S. Effect of ascorbic acid on the monosodium glutamate-induced neurobehavioral changes in periadolescent rats. Bratislava Med J. 111:170-5, 2010.

15. Hajiani M, Golestani A, Shariftabrizi A, Rastegar R, Payabvash S, Hassanzadeh Salmasi A, et al. Dose-dependent modulation of systemic lipid peroxidation and activity of anti-oxidant enzymes by vitamin E in the rat. Redox Rep. 13:60-6, 2008.

16. Aksu I, Baykara B, Ozbal S, Cetin F, Sisman AR, Dayi A, et al. Maternal treadmill exercise during pregnancy decreases anxiety and increases prefrontal cortex VEGF and BDNF levels of rat pups in early and late periods of life. Neurosci Lett. 516:221-5, 2012.

17. Arai K, Matsuki N, Ikegaya Y, Nishiyama N. Deterioration of spatial learning performances in lipopolysaccharide-treated mice. Jpn $J$ Pharmacol. 3:195- 201, 2001.

18. Casadesus G, Milliken EL, Webber KM, Bowen RL, Lei $Z$, Rao $C V$, et al. Increases in luteinizing hormone are associated with declines in cognitive performance. Mol Cell Endocrinol. 269:107-11, 2007. 
19. Bancroft JD, Gamble M. Theory and practice of histological techniques. Elsevier health sciences; 2008.

20. Happerfield LC, Bobrow LG, Bains R, Miller KD. Peroxidase labelling immunocytochemistry: a comparison of eleven commercially-available avidin-biotin systems. Br J Biomed Sci. 50:21-6, 1993.

\section{Li S, Reinprecht I, Fahnestock M, Racine}

RJ. Activity-dependent changes in synaptophysin immunoreactivity in hippocampus, piriform cortex, and entorhinal cortex of the rat. Neuroscience. 115: 1221-9, 2002.

22. Sreejesh PG, Sreekumaran E. Effect of monosodium glutamate on striatohippocampal acetylcholinesterase level in the brain of male Wistar albino rats and its implications on learning and memory during aging. Biosci Biotech Res Comm. 11:76-82, 2018

23. Hassaan PS, Dief AE, Zeitoun TM, Baraka AM, Deacon RMJ, Elshorbagy A. Cortical tau burden and behavioural dysfunctions in mice exposed to monosodium glutamate in early life. PLoS One. 14;14(8):e0220720, 2019.

\section{Abdul-Hamid M, Galaly SR, Ahmed RR,} Hamdalla HM. Monosodium Glutamate as a Food Additive: Toxic Implications and the Protective Role of Quercetin. Merit Res. J. Med. Med. Sci. 5:384-402,02017.

\section{Umukoro S, Oluwole GO, Olamijowon} HE, Omogbiya AI, Eduviere AT. Effect of monosodium glutamate on behavioral phenotypes, biomarkers of oxidative stress in brain tissues and liver enzymes in mice. World J Neurosci. 5:339, 2015.
26. El Agamy DF, Naguib YM. CoQ10 ameliorates monosodium glutamate-induced alteration in detrusor activity and responsiveness in rats via anti-inflammatory, anti-oxidant and channel inhibiting mechanisms. BMC Urol. 19:103, 2019.

27. Onaolapo AY, Odetunde I, Akintola AS, Ogundeji MO, Ajao A, Obelawo AY, et al. Dietary composition modulates impact of food-added monosodium glutamate on behaviour, metabolic status and cerebral cortical morphology in mice. Biomed Pharmacother. 109:417-28, 2019.

28. Uchida K. 4-Hydroxy-2-nonenal: a product and mediator of oxidative stress. Prog Lipid Res. 42: 318-43, 2003.

29. Mattson MP. Glutamate and neurotrophic factors in neuronal plasticity and disease. Ann N Y Acad Sci. 1144:97-112, 2008.

30. Fujikawa DG. Prolonged seizures and cellular injury: understanding the connection. Epilepsy Behav. 7:3-11, 2005.

31. Ilhan A, Gurel A, Armutcu F, Kamisli S, Iraz M. Antiepileptogenic and antioxidant effects of Nigella sativa oil against pentylenetetrazol-induced kindling in mice. Neuropharmacology. 49:456-64, 2005.

32. Farombi EO, Onyema OO. Monosodium glutamate-induced oxidative damage and genotoxicity in the rat: modulatory role of vitamin $\mathrm{C}$, vitamin $\mathrm{E}$ and quercetin. Hum Exp Toxicol. 25: 251-9, 2006.

33. Hussein U, Hassan N, Elhalwagy M, Zaki A, Abubakr H, Nagulapalli Venkata K, et al. Ginger and propolis exert neuroprotective effects against monosodium glutamate-induced neurotoxicity in rats. Molecules. 2:1928, 2017. 
34. Cervantes MCR, Jarero JJ, Castillo JM, Lopez AGM, Martinez YG, Martínez SC, et al. The recombinant human erythropoietin administered in neonatal rats after excitotoxic damage, induces molecular changes in the hippocampus. Front Neurosci. 13:118, 2019.

35. Sharma A, Kalotra S, Bajaj P, Singh H, Kaur G. Butanol Extract of Tinospora cordifolia Ameliorates Cognitive Deficits Associated with Glutamate-Induced Excitotoxicity: A Mechanistic Study Using Hippocampal Neurons. Neuromolecular Med. 1-19, 2019.

36. Schmitt U, Tanimoto N, Seeliger M, Schaeffel F, Leube RE. Detection of behavioral alterations and learning deficits in mice lacking synaptophysin. Neuroscience. 162:234-43, 2009.

37. El-Tantawi GH, and El-Namshan. MM Monosodium glutamate followed by barley in mice: A histological, immunohistochemical and ultrastructural approach. Egypt J Exp Biol. 11:81-93, 2015.

38. El-Gohari KMA, Bahei-Eldin IA, Habib EMK, Saad SA, Rady HYS, Said AMA. Neuroprotection of the rat's retinal ganglion cells against glutamate-induced toxicity. $J$ Egypt Ophthalmol Soc. 109:135-144, 2016.

39. Nakamura R, Kurita K, Kawanami T, Kato T. An immunohistochemical study of Purkinje cells in a case of hereditary cerebellar cortical atrophy. Acta Neuropathol. 97:196-200, 1999.
40. Sasaki-Hamada S, Hojo Y, Koyama H, Otsuka H, Oka J. Changes in hippocampal synaptic functions and protein expression in monosodium glutamate-treated obese mice during development of glucose intolerance. Eur J Neurosci. 41:1393-1401, 2015.

41. Soliman GF, Khattab AA, Habil MR. Experimental Comparative Study of potential anxiolytic effect of Vitamin $\mathrm{C}$ and Buspirone in rats. Funct Foods Heal Dis. 8:96-106, 2018.

42. Shivasharan BD, Nagakannan P, Thippeswamy BS, Veerapur VP. Protective effect of Calendula officinalis L. flowers against monosodium glutamate induced oxidative stress and excitotoxic brain damage in rats. Indian J Clin Biochem. 28:292-8, 2013.

43. Ibrahim MA, Buhari GO, Aliyu AB, Yunusa I, Bisalla M. Amelioration of monosodium glutamate-induced hepatotoxicity by vitamin C. Eur J Sci Res. 60:159-65, 2011.

44. Foda A, Ibrahim E-S, Gouida M, Elsherbiny E. Hepato-Protective Effect of Vitamin C Extract from Kiwi Fruit on Hepatic Injury Induced by Monosodium Glutamate. SCVMJ. 24:27-36, 2019.

45. Calis IU, Cosan DT, Saydam F, Kolac UK, Soyocak A, Kurt H, et al. The effects of monosodium glutamate and tannic acid on adult rats. Iran Red Crescent Med J. 18(10), 2016.

46. Eid RA, Dallak M, Al-Shraim M, Ellatif MA, Al-Ani R, Kamar SS, et al. Suppression of Monosodium GlutamateInduced Acute Kidney Injury and Renal Ultrastructural Damage in Rats by Vitamin E. Int J Morphol. 37:1335-1341, 2019. 
47. Hansen S, Tveden-Nyborg P, Lykkesfeldt J. Does vitamin C deficiency affect cognitive development and function? Nutrients. 6:3818-46, 2014.

48. Muley BP, Khadabadi SS, Banarase NB. Phytochemical constituents and pharmacological activities of Calendula officinalis Linn (Asteraceae): a review. Trop J Pharm Res. 8:455:465, 2009.

49. Ricciarelli R, Argellati F, Pronzato MA, Domenicotti C. Vitamin E and neurodegenerative diseases. Mol Aspects Med. 28:591-606, 2007.

50. Hashem HE, Safwat MDE-D, Algaidi S. The effect of monosodium glutamate on the cerebellar cortex of male albino rats and the protective role of vitamin $\mathrm{C}$ (histological and immunohistochemical study). $J \mathrm{Mol}$ Histol. 43:179-86, 2012.

\section{Ibrahim AA-S, Mohammed HO, Hassan}

NH. Effect Of Monosodium Glutamate On The Cerebellar Cortex Of Male Albino Rat And Protective Role Of Vitamin C. Zagazig Univ Med J. 25:250-60, 2019.

52. Paul MVS, Abhilash M, Varghese M V, Alex M, Harikumaran Nair R. Protective effects of $\alpha$-tocopherol against oxidative stress related to nephrotoxicity by monosodium glutamate in rats. Toxicol Mech Methods. 22:625-30, 2012.

\section{Nam SM, Seo M, Seo J-S, Rhim H, Nahm}

S-S, Cho I-H, et al. Ascorbic acid mitigates D-galactose-induced brain aging by increasing hippocampal neurogenesis and improving memory function. Nutrients. 11:176, 2019.
54. Ambrogini P, Minelli A, Galati C, Betti M, Lattanzi D, Ciffolilli S, et al. Postseizure $\alpha$-tocopherol treatment decreases neuroinflammation and neuronal degeneration induced by status epilepticus in rat hippocampus. Mol Neurobiol. 50:24656, 2014.

55. Rendón-Ramírez A-L, Maldonado-Vega M, Quintanar-Escorza M-A, Hernández G, Arévalo-Rivas B-I, Zentella-Dehesa A, et al. Effect of vitamin $\mathrm{E}$ and $\mathrm{C}$ supplementation on oxidative damage and total antioxidant capacity in lead-exposed workers. Environ Toxicol Pharmacol. 37:45-54, 2014.

56. Grodstein F, Chen J, Willett WC. Highdose antioxidant supplements and cognitive function in community-dwelling elderly women. Am J Clin Nutr. 77:975-84, 2003.

57. Navarro-Yepes J, Zavala-Flores $\mathbf{L}$, Anandhan A, Wang F, Skotak M, Chandra N, et al. Antioxidant gene therapy against neuronal cell death. Pharmacol Ther. 142: 206-30, 2014.

58. Shukitt-Hale B. The effects of aging and oxidative stress on psychomotor and cognitive behavior. Age (Omaha). 22:9-17, 1999. 